\title{
CONCERNING THE VIRTUAL IN THE REAL
}

\author{
Niklas Damiris ${ }^{1}$ and Helga Wild ${ }^{2}$ \\ 1) Visiting Scholar, Stanford University, USA \\ 2) Institute for Research on Learning, Menlo Park, USA
}

\begin{abstract}
Global communication technologies need to go beyond intersubjective symbolic exchange toward supporting the engagement of people in their cultural context. Hence, what is needed is an understanding for the way in which the efficacy of cultural contexts manifests itself.

Mass media have been successful in including people in a cycle of cultural production and consumption, but this process is accompanied by increased standardisation. The interindividual forms of communication, on the other hand, lend themselves to customisation but are too text-based to leverage the cultural context for purposes of creative collaboration.

Virtual reality, we claim, by propagating a form of interaction based on immersion, could become the means of addressing and operating with cultural contexts. But then it needs to comprehend their ontological status in a new way.

We place the efficacy of the cultural in the domain of the virtual. Our short paper gradually develops and refines a notion of virtuality that will stand up to the demands of grounding a development in that direction. In the process we draw on philosophy, cultural and media studies, and psychoanalysis.
\end{abstract}

\section{INTRODUCTION}

As the fin de siecle is approaching and experts and laymen alike feel the need to adjust their world-view after the turbulent changes of this century, there is much talk about globalisation and little doubt that computers are a major driving force in that trend. Globalisation is a multifaceted syndrome that can be described as the collection of practices and processes which increasingly overcome geographical separation and socio-economic and cultural differences.

The entire phenomenon can and has been explained as the effect of purely economic factors: capitalism writ large. (Damiris 1997, Wallerstein 1995). Wallerstein's world-system hypothesis, whose claim is that the globe is becoming a single economic system ${ }^{1}$, implies that the dynamics of its development are largely internal; it is materially self-contained and sufficient. However, this new phenomenon of geosystemic integration should also be approached in terms of the aforementioned forces of computer mediation, from the perspective of the globalisation of communication. 
If the world is becoming interconnected on a global scale, communication needs to be able to span vast geographical and social distances, something that has been made possible by the ubiquity or near-ubiquity of media technologies. On the one hand, interpersonal mediated communication has taken the form of mail, email, fax, telex and phone, all of them mainly verbal and text-based and very effective as far as communication as message exchange or transmission goes. Mass media, on the other hand, have engendered and perfected large-scale broadcasting but also brought about a standardisation which is not always making the most of the human capacity for communication. Information, in general, has become synonymous with the transmission and decoding of symbolic messages.

The globalisation of communication promises an unfolding of the riches of the earth's cultural, historical and semiotic materials. But, in order to make good on such promise and leverage the diversity it is giving us access to, information transfer and processing is not good enough. Take as an example the case of a written message: it does not easily convey the context within which it is produced, and yet it is usually the local context of its engendering that holds the creative potential. So, clearly, if one wants to carry forward or build on the potential immanent in the local, the interactions and relations between the concerned parties must take the form of an engagement with one another and of involvement in each other's cultural and social surround -in short they require a form of immersion.

This is what face-to-face contact used to provide and guarantee. But face-toface contact is precisely what is difficult to maintain in the age of transnational corporations; remoteness from one's collaborators, whether customers or colleagues, is the order of the day. (That, however, can become a source of creative advantage! The important and potentially beneficial difference between face-to-face and remote contact is that whereas face-toface creates one unifying context for all participants, remote contact could both preserve and then leverage the multiplicity of contexts if it succeeds. Admittedly, a tall order!)

But, let's return to the concern with cultural immersion which such globalised communication ought to afford. Immersion brings to mind right away the efforts at constructing experiences in virtual reality environments. Virtual reality is at the moment merely a rarified construct accessible to a few researchers and techno-insiders. But if appropriately linked with the expanding domain of a computer-mediated and supported intercultural communication it could be developed to express a new mode of contact that bridges both social and physical distance while allowing people to participate in each other's cultural environment.

So, we are imagining a kind of virtual reality which does not yet exist. In trying to contribute to its development and most ethical use, we need to come to terms with the notion of virtuality in general. So far the often heated debate on virtual reality revolves around the old metaphysical conundrum of illusion and reality, with one side upholding the illusory 
underpinnings of the real and the other defending an unduly positivistic form of realism. By construing the relation as an opposition both sides, the defenders of the real, and the advocates of the virtual, if we may so label them, act as if they already know what virtual is and means. And this closes the door to a potentially promising development in mediatisation. The gain we expect from studying virtuality sui generis is insight into the underlying conditions of the human life-world which can thus guide our steps in this territory ahead.

\section{VIRTUALITY IS NOT IN OPPOSITION TO THE REAL}

For Technologists virtual reality (VR) points to several registers. Some are talking about pieces of interface hardware, e.g. head-mounted displays, datagloves and eyephones, others speak about applications, e.g. medical imaging, remote surgery, or of the cultural landscape emerging around the use of VR technology, cyberspace and cyberfiction. Today VR technology systems are rationalised by the drive to produce an all inclusive immersive sensory illusion, the illusion of being present in another place, a virtual reality.

On the other hand, Jaron Lanier who coined the term speaks of VR as leading to a mode of post-symbolic communication and affording us therefore unmediated subjective expression. The concern with the very status and aim of virtual reality is prefigured in the polemic between photography and painting in the 1860's. The mechanical process of recording reflected light onto a sensitive surface had been welcomed as a safeguard against intentional manipulation of representations. One could easily tell the difference between the mechanically generated, unmitigated image produced by the camera and a painting of the identical subject matter. The photographic image brought the bias intrinsic to a painting to the fore and forced painting to admit that it was an articulation of an experience rather than the objective depiction of reality.

The situation is a bit different with computers. Digital technology can simulate existing media to the extent of being visually indistinguishable from them. As long as the criterion for verisimilitude is based on visual appearances, the boundaries separating different media and production processes are blurred rather than sharpened. Once the image is stored as numeric data in computer memory it can be compressed and processed in unlimited ways without physical degradation and without any trace of the intervening manipulations being preserved in the final product. The mechanical mode of production increases the possibilities of intervention and manipulation, and prior knowledge of an image's or text's history, its source, mode of production and reproduction become necessary and relevant factors in accurately grasping the significance of the digital image and text. Realism is then - as postmodern theorists have nastily, but irrefutably put it - an effect produced by the coinciding of the image or representation with 
that which a particular social group posits and presumes to be real. This means, reality is not how the world 'really' is, but the ground zero that every society needs in order to perceive. Furthermore mediatisation is not defined in opposition to a non-mediated reality, but - like the proverbial turtles - it reaches all the way down.

This is where the postmodern debate around media connects up with the older philosophical discussions about the status of illusion: whether one can even speak of artificiality when illusions are engendered by natural phenomena and are therefore as natural as nature itself.

Media have always sought to utilise and refine naturally occurring perceptual illusions. One could go so far as consider the evolution especially of visual media from the development of perspective, photography and dioramas to moving pictures and wide-screen cinema as the progress of illusion. What is worth noting about this obsession with illusion is the drive toward ever more encompassing ones, the drive to fool the mind into feeling confronted with reality when the objective conditions are not actually given.

Illusion, in this reading, seems to be saddled with the Sisyphean task of approximating the real with ever greater precision until it can substitute for it. But this polarisation of illusion and reality potentially obscures their complementary roles in life. When the relation of illusion and reality is construed as opposition, it forces one to take virtuality to be nothing but a simulacrum, a copy of the real.

\section{PART OF THE THIRD WORLD}

Popper's simple but helpful 'three world's schema' may help us escape this predicament. He has suggested a tripartite differentiation of the life-world: world one contains the physical, world two is the realm of mental states, and world three contains the products of the human mind, concepts, constructs and images. ${ }^{2}$ No matter how one might feel about the hierarchy he sets up among these worlds, what is important is that he grants world three parity with the other two.

VR, according to this partitioning, is a part of world three and shares its unique mode of existence. This mode is not to be compared or juxtaposed to the existence (and reality) of objects in world one or two, but has to be taken on its own terms. If the earlier approach made the constructs of the human mind out to usurp or reproduce the domain of the real, in Popper's we deal with entities with different modes of existence that jointly make up the real.

Historically, of course, the different worlds can stand at times in conflict with one another, but such a relation can not put in question their ontological autonomy. For example, at present under the impact of mass media and global information exchange it looks like world three begins to 
edge out world one. With TV, advertisement and news we are increasingly inhabiting an umwelt made up by the products of our collective intelligence.

The relevance of Popper's three-world schema is that we can look at world three as a continent populated by a vast collection of characters and materials with their own cultural - i.e. fictional, narrative, semiotic efficacy, what Latour called actant. ${ }^{3}$ Instead of the wasteland that we normally associate with the debris of a mass media culture we get a glimpse of something very different: unexplored areas in formation (in-formation, the very idea!). But what is the ontological status of these world fragments? What is their reality? Clearly they exist in a realm other than the actual. Philosophers already have come up with a number of candidates to account for them: Aristotelian potentia, Platonic forms, fictionality in literature, Leibnizean possible worlds, and more. We want to promote the virtual as yet another strong candidate.

\section{OTHER THAN POTENTIALITY}

Aristotle accounts for reality via the dynamic relation between actuality and potentiality. Actuality is what at any given moment makes up reality; and potentiality is at its service. The Aristotelian account of their interrelation puts the emphasis on the act of the soul which brings about the fullest, most 'real' and complete form of being, that is, actuality. It does this by conforming matter to thought, that is, nous. Potentiality, by comparison, is mere pre-formation and therefore an incomplete and inadequate form of existence.

At first glance, the virtual could be seen as analogous to the powers (potentia) of the soul along with sensibility, emotions, and thought. Potentialities of this kind permit the soul to know and think and move by actualising matter and giving it definite form always in accord with the potential involved. The being of the potential is then dependent on the being of the soul which is destined to know and to move. So far, so good.

It turns out to be impossible, however, to imagine what matter and what form the virtual could evoke in the world, to say how it can become subject to an act of the soul. Sensibility is a potentiality; its actuality is sensation (the form), which is at the same time the realisation of the sensible in the object (its matter). Mutatis mutandis, if virtuality is a potentiality in the same sense, what would correspond to its formal and what to its material expression in actualisation? One can not think what the virtual's equivalent to sensation and the sensible would be.

The virtual is also essentially different from the possible understood as something that might or might not be, something that is neither necessary nor impossible. Kirkegaard maintained that the possible does not become necessary just because it is realised, but rather remains possible. Walter Benjamin similarly cautioned against the tendency to identify the real with the actual, in his example, with what conquers and triumphs in the world: 
the history of the world should not be thought of as the history of the winners. ${ }^{4}$ The difference is that the virtual need not become realised in order to be efficacious.

The metaphysics of mass media shows surprising congruity with Aristotle's identification of actuality with reality: their condition of presence might indeed be viewed as the culmination of a bias against potentiality that originates precisely with Aristotle. The assertion of the primacy of actuality over potentiality, consigns to irrelevance or second class status all that is implicate, tacit and subterranean.

\section{PRESENCE IN MASS MEDIA}

What Aristotle called actuality is in the age of mass media the result of the workings of a large social and technical machinery. Think of the elaborate production processes of such media as print, film, TV, and advertising. Characteristically, their various products aim to reach simultaneously and as fast as possible the largest number of people. In contemporary society then the dominant form and content of communicative production become necessarily standardised and the same goes for their reception: instant experience, ready-made and canned like instant coffee. This contributes to the homogenisation of the social imaginary. Processes of media production and reception supplant the acts of the soul. But we are still dealing with the dynamic transformation of matter into form, largely without conscious awareness. This time, though, the potentia resides in supra-individual apparatuses and the act of producing reality is a distributed collective achievement.

The processes at work in mass media have been accurately likened to the mechanisms in dream-work described by psychoanalysis. The dream and the dream mechanisms - condensation, displacement, symbolism, modification of affective states and secondary elaboration - provide us with a framework for understanding the manipulation of contents as well as minds by the mass media.

Dream-work entails subtle logical and semantic operations on the dream content and its product is a form of presence which is comparable to the mass media-produced instant-experience: a shifting boundary between past and future, a res fluens which is perishable and quickly vanishes.

Presence in either case is not the experience of the present but, quite the reverse, that of its lack, its insubstantiality. Nor has presence anything to do with what is new: the stuff of mass media just like those of dreams feeds on leftovers, cast-offs and debris, granted a makeover under the pressures of the moment. Presence comes with an impression of deja vu, tedium, which is liable to tip over into emotional exaggeration and to spawn intolerance, if not fanaticism. 
The standardisation along a single register of all the messages and all the commodities of mass culture, the melting down of major distinctions, the confusion of differences of degree and kind, are not due, as many proclaim, to the democratic character of the culture industry, to its overly ambitious effort to reach everybody, but to the totalitarian character of 'presence', to the way it intimidates everyone into assimilating instantly, what it serves up. Similarly, the eclectic instantaneity of the dream which, in Freud's view, erases all differences and juxtaposes the most disparate of entities, proceeds in analogous fashion creating a horizon in which the only thing that stands out is what appears on that instant's stage.

\section{VIRTUALITY AS CULTURAL EFFICACY}

The positive side of this kind of presence is that it actively reaches into the multiplicity of immanent sedimented characters and structures which we located earlier in Popper's third world. Underneath mass media's prefabricated effects we can sense an original realm of qualitative differentiations which lie at the heart of the success of mass media. Not unlike the reserve of fossils that turned into oil and thus potential fuel for us, this realm can fuel production and action. But it does so not in a causally efficacious manner nor does it work haphazardly.

Here is the way to imagine the action of the virtual. Christian theology distinguishes actual intention from virtual intention. The actual intention is what the priest has in mind at the moment of celebrating mass, preparing and offering communion. The virtual intention is what inheres in these acts in virtue of their constituting a religious ceremony, even if the priest does not have the same intention present to his mind as he carries out the necessary actions.

This use of the term 'virtual' is significant because it confers on virtuality an efficacy that does not have to go through the actual. The virtual has a degree of stability that is independent of consciousness. Unlike the potential and the possible which are always dependent on their realisation in the actual and on the act of the soul, the virtual embodies a different reality, outside the actual, external to the subject. Virtuality, we conclude, is the domain of the force of the culture which lends cultural expressions their binding power and forms the horizon against which presence gets produced and the actual gets interpreted. In short, the virtual bespeaks the efficacy of things that have no causal efficacy.

To virtualise the world is not to double it but, on the contrary, to complicate it, to release other configurations and paths in it. However, the ordinary way to increase complexity is to abstract from actuality, by making something more abstract and general. Such increase in abstraction is followed by an inverse reduction in actual detail. The ruse of this reduction is to make it seem that outside the aegis of the specifiable all is ineffable, pure disorder and anarchy. The result is that formalisation and ordering which usually 
accompany abstraction appear coextensive with the actual in what otherwise feels like a nebulous realm.

The virtual on the other hand complicates differently precisely because its efficacy is exercised but not at the expense of the actual. Attending to the virtual intensifies our contact with actuality. The virtual is, as Deleuze so eloquently puts it, "real without being actual; ideal without being abstract!"5

\section{VIRTUALITY AS SUSTAINING MULTIPLICITY}

We owe the approach to virtuality as sustaining multiplicity to Gilles Deleuze and Felix Guattari, the two contemporary thinkers who understood its importance in the human life-world. Guattari writes, not in the voice of the detached philosopher, but in the voice of the schizophrenic who is less prone to follow pre-fabricated concepts and therefore more open to what surrounds presence, "A cry, a monochrome blue, makes an incorporeal, intensive, non-discursive, pathic Universe suddenly appear, and as a result other Universes, other registers, other machinic bifurcations are brought about: singular constellations of universes." 6 This movement traces the sensation back to the virtual realm which not only surrounds it with the penumbra of a whole universe and in turn it 'gives itself', but moreover is followed by a whole cornucopia of universes. If one interprets the first universe as the cultural context in which the sensation lies embedded, the constellation of universes that follows has to be seen as a multiplicity of other cultural contexts equally, though less directly, related to the same actuality.

Admittedly being inspired by the schizophrenic condition may seem perverse to a rationalist, or simply irrelevant to a technologist. But if our suggestion, that VR holds promise as an alternative way for deploying and designing communication technologies is heeded, then what Guattari observed of schizophrenics vis a vis the virtual, could be said of the different cultural contexts' relation to it. In both cases we are describing a mode of relating to virtuality that reveals its involvement with the actual without being of it.

Take as an example the case of a symphony concert. Its cultural context is an assemblage of elements bound together to create this musical event. It is a dynamic magma, composed of such heterogeneous components such as musicians, their instruments, the score, the space of the concert hall, dress code, rules for proper conduct, to mention only the most obvious ones. Less obvious factors include: the musical tradition inspiring the particular piece, the experience and training of both the musicians and their audience, musicological analysis, the occasion for the performance and so on.

Currently broadcasting media have a big influence on how the cultural context of the symphony concert (which is what is happening not merely its 
background) is delivered and experienced. Ironically, today's information technology has even less impact on this cultural context than mass media. Admittedly, a CD-ROM on classical musical performances can make a lot of background history available to those interested; also having a VCR and a CAM-corder and hooking both to your hi-definition TV, which is also your computer that runs software for sound-mixing, puts quite a bit more choice and control in how the actuality occasioned by the concert performance is made present to individuals. Still, there is little practical awareness or available media technology for engaging directly the cultural context in which the performance is broadcasted, recorded or edited and is only the tip of the iceberg.

The same can be said about the interpersonal uses of information technology. Most people, aside from hard-core lovers of technology, feel that they are missing out on something which the abundance of information does not get to, but they have a hard time specifying what it is. The excessive, but limited use of the Internet bears witness to that.

Often the discussion drifts into issues of authenticity, of the aura of live presence when one attends in person, of a feeling of being part of a 'scene' and so on. All of the above admittedly are symptomatic. But by themselves they are neither a remedy nor what has to be captured by technology aimed at simulating the feeling of being there. Alas, what is yearned after can't be simulated for it is not an actuality in the first place. It is the transitory outcome and piece of a process always in flux. So the challenge here is not to simulate the wave, so to speak, but to surf on it.

\section{IN CONCLUSION}

Communication technology should not aim at the total replication of the experience qua information. If it were to succeed at that the result would be psychotic breakdown due to over-stimulation. The richness of reality translated into something that can be delivered through data channels becomes unbearable and the only reasonable response is for the senses to shut down.

The alternative way to recreating reality lies in abstracting away from local specificity to a higher plane of generality and transmitting this more abstract version. The work of abstraction has to be performed by selection mechanisms firmly put in place, but the unsolved question is how the selection criteria can respond to the local context.

You will recall that we have encountered each of these moves earlier, though in slightly different rendition: the first is the move to approach reality asymptotically which comes from conceptualising the problem as an opposition between illusion and reality; the other is the move to load potentia by abstracting from actuality and then to leave it up to the new cultural context to realise it. Both moves are misguided because they ignore 
the role of the virtual and therefore misconstrue the ontology that is at work in communication across cultural contexts.

This paper, then, reaches a negative conclusion, but we feel this is an important result, nevertheless. It tells us that the approaches taken by information technology to overcome physical and social distances are bound to fail because they are based on the wrong conceptual foundations. The right choice, we feel, relates the actual to the virtual in the mode we have outlined.

\section{NOTES}

1 Wallerstein, Immanuel; 1995. p.13.

2 Popper, 1979, p.154.

3 Actant is the term used by Latour and Serres to denote entities with causal efficacy other than the human. Latour and Serres, 1995.

4 Benjamin, 1969.

$5 \quad$ Deleuze, 1988 , pg 55.

$6 \quad$ Guattari, 1995, pg 96

\section{REFERENCES}

Benjamin, W. (1969) Theses on History. Illuminations. Schocken, NY.

Castoriadis, C. (1997) World in Fragments. Writings on politics, society, psychoanalysis, and the imagination. Stanford University Press.

Damiris, N. and Wild, H. (1997) Corporations and the globalization blues. Seminar talk and paper delivered at ProjectCentre CESAR, Copenhagen Business School, Denmark, November 1997.

Deleuze, G. (1988) Bergsonism. Zone Books.

Guattari, F. (1995) Chaosmosis. an esthetico-aesthetic paradigm. Indiana University Press.

Popper, K. (1979) Objective knowledge. an evolutionary approach. Oxford Press.

Serres, M. and Latour, B. (1995) Conversations on science, culture, and time. University of Michigan Press.

Wallerstein, I. (1995) Unthinking social science. the limits of nineteenth-century paradigms. Polity Press.

\section{We dedicate this paper to the memory of our friend Cornelius Castoriadis}

